\title{
ACUPUNTURA, MEDICINA ANCESTRAL PARA TRATAMIENTO DE LAS ENFERMEDADES
}

\author{
Ángel Chú Lee ${ }^{1}$, Lina Barreto Huilcapi ${ }^{1}$, Brígida Agudo Gonzabay ${ }^{1}$ \\ Emerson Maldonado Guerrero ${ }^{1}$, Sixto Chiliquinga Villacís ${ }^{1}$ \\ Universidad Técnica de Machala (Ecuador) ${ }^{1}$ \\ achu@utmachala.edu.ec
}

Cómo citar este artículo: Chu Lee, A., Barreto, L., Agudo, B., Maldonado, E. y Chiliquinga, S. (2015) Acupuntura, medicina ancestral para el tratamiento de las enfermedades. CUMBRES, Revista Científica. 1(1) 43 - 47

\section{RESUMEN}

La civilización china con más de 5000 años de historia, nos dejó cuatro grandes inventos para la humanidad: la brújula, el papel, el ábaco y la pólvora, pero también para el cuidado de la salud, un gran tesoro de la medicina tradicional china como es la acupuntura.

Se lo define a la acupuntura como un arte y una ciencia que consiste en inserción de agujas finísimas (descartables) sobre puntos energéticos en la superficie del cuerpo para restituir o equilibrar el flujo de energía en líneas invisibles del cuerpo, llamados meridianos. En término occidental, la inserción de agujas activan pequeñas fibras mielinizadas en el músculo estriado, estos envían impulsos al Sistema Nervioso Central produciendo liberación de Neuropéptidos (Endorfinas, Encefalinas, Dinorfinas) bloqueando los mensajes dolorosos procedentes de cualquier parte del cuerpo.

Palabras clave: Acupuntura, endorfinas, meridianos.

\section{ABSTRACT}

The Chinese civilization with more than 5000 years of history, we left four great inventions for mankind: the compass, paper, the Abaco and gunpowder, but, also for the health care a great treasure of Chinese traditional medicine as is acupuncture.
It is defined to acupuncture as an art and a science that involves inserting very fine needles (disposable) on energy points on the body surface to restore or balance the flow of energy in invisible lines of the body, called meridians. In west end, the insertion of needles activate small myelinated fibers in the striated muscle, these send impulses to the Central Nervous System producing release of neuropeptides (endorphin, enkephalin, Dinorfinas) blocking the painful messages from any part of the body.

Keywords: Acupuncture, endorphins, meridians.

\section{INTRODUCCIÓN}

Desde el origen de los tiempos, junto con la humanidad aparecieron las enfermedades, el hombre primitivo tratando siempre de mitigar sus dolencias. En la época de piedra, (período neolítico 2500 A.C.) se usaban las BIAN, o agujas de piedra, con propósitos curativos. Esto constituye la base más rudimentaria de la acupuntura. Cuando la humanidad entra a la edad de Bronce y en la edad de Hierro, se empezó a fabricar agujas metálicas en lugar de las de piedra. Es decir, el material de las agujas fueron fabricándose de acuerdo a la época.

El texto más antiguo de la medicina China es HUANGDI NEIJING (Canon de Medicina Interna del Emperador Amarillo), compilado entre 500 - 300 A.C. Es un compendio de las experiencias médicas donde se describe la teorías básicas de la medicina tradicional china tales 
como yin -yang, los cinco elementos, zang-fu (órganos), los canales y colaterales, qí (energía vital) y xue (sangre) etiología, patología, métodos de diagnóstico y diferenciación de síndromes, así, como los conocimientos fundamentales sobre puntos de acupuntura y métodos de inserción de las agujas.

En el siglo VI, la acupuntura fue introducida en Corea y Japón, donde se convirtió en parte integral del sistema de salud de cada país. A partir de allí, hizo su camino hacia el sur de la India y hacia países del sudeste asiático como Vietnam. Gracias al efecto positivo en la salud de muchas personas, especialmente los de ascendencia real y de influyente posición política, la acupuntura impactó, exitosamente, todo el continente asiático.

A principios del siglo XX la acupuntura llega a Europa traída por Solie de Mourant a Francia de donde se expande a diversos países europeos, estableciendo diversas escuelas para enseñanza y aprendizaje de la acupuntura (Soulié de Morant, 1990).

Con el establecimiento de la República Popular China en 1.949 se promueve el estudio y la investigación de la Medicina Tradicional publicándose más de 9000 artículos sobre la acupuntura y se tratan más de 300 enfermedades, lográndose gran éxito en más de 100. En 1958 se realiza la primera operación quirúrgica bajo anestesia acupuntural y en 1968 sobre la base de la neurofisiología moderna, se practica la cráneo acupuntura.

En occidente se desarrolla la acupuntura auricular por el Francés (Nogier, 1954), el Dr. Reinhold Voll de Alemania establece su electro-acupuntura (electro-acupuntura de Voll) para el diagnóstico y tratamiento de frecuencia con mediciones electrónicas (Voll, et al., 1978.).

Se emplean los Rayos Láser en los puntos acupunturales y se está dando paso a la nueva investigación, diagnóstico y terapéuticas usando sistema de computación.

\section{Mecanismo fisiológico de la acupuntura}

El mecanismo de acción de la acupuntura se fundamenta en tres niveles importantes:

\section{Liberación de neuropéptidos - endorfinas}

Se trata de neuro moduladores que intervienen en los mecanismos de control del dolor del propio cuerpo. La información dolorosa, que llega al cerebro por vías ascendentes, es regulada por diferentes sistemas descendentes
(Opioide, Noradrenérgico, Serotoninérgico). Las endorfinas son neurotransmisores Opioides «endógenos», que regulan la percepción del dolor. La estimulación de ciertos puntos de acupuntura inicia una cascada biológica que determina una masiva liberación de opioides endógenos, modulando y controlando el dolor (Goldberg, 1999).

Con las nuevas investigaciones que se están realizando se conoce que la acupuntura produce un incremento de los niveles de neuropéptidos opioides endógenos modificando la percepción dolorosa, en el asta posterior de la médula espinal, en la sustancia gelatinosa de Rolando, la transmisión de la información nociceptiva se modula mediante mecanismos encefalinérgicos, existiendo encefalinas en las sinapsis de las neuronas de la sustancia gelatinosa que pueden modular la transmisión de la sensibilidad nociceptiva y actúan tanto en las sinapsis aferentes primarias como en las terminales postsinápticas. La acupuntura está muy vinculada a estos mecanismos.

Los neuropéptidos endógenos (B-endorfinas, encefalinas, dinorfinas, etc. ) son liberadas por medio de la electro acupuntura en dependencia de la frecuencia de estimulación, a bajas frecuencias (2-4 Hz) se liberan las B-endorfinas y a altas frecuencias $(100 \mathrm{~Hz}$ ) las Dinorfinas, que interactúan a nivel de la corteza cerebral y la médula espinal, igual planteamiento hace que las encefalinas son liberadas a diferentes frecuencias de estimulación, a $2 \mathrm{~Hz}$ las B-endorfinas y a $100 \mathrm{~Hz}$ las Dinorfinas, destacando la importancia del líquido cefalorraquídeo en los efectos analgésicos de la acupuntura por las sustancias liberadas en él, además, la resonancia magnética nuclear ha reflejado relación entre la estimulación de determinados puntos de acupuntura y zonas de la corteza cerebral, lo que abre la posibilidad de nuevos estudios científicos (Cabana, et al., 2004).

A nivel del Sistema Nervioso Central, las neuronas endorfinérgicas colaboran en el procesamiento cerebral del estímulo doloroso, las endorfinas alteran la percepción doIorosa en la médula espinal, el mesencéfalo, el tálamo y la corteza cerebral, por lo que desempeñan una importante función en la analgesia asociada a la acupuntura.

\section{Teoría de la compuerta de Melzack y Wall (1969) (GATE CONTROL)}

La teoría de la compuerta se refiere a la incapacidad del cerebro de procesar simultáneamente más de una percepción o estímulo (sea o no doloroso) (Melzack, et al., 1965).

De este modo la acupuntura, mediante un estímulo en la piel, genera información que «compite» con el dolor origi- 
nal logrando desplazar a este del procesamiento central. Esto se podría comparar con el hecho de que un dolor tapa a otro dolor», de la misma manera que lo hace una distracción (de intensidad adecuada). De ésta forma y progresivamente el «mensaje original doloroso» es opacado y muchas veces anulado por el estímulo indoloro producido en la piel por las agujas de acupuntura. De acuerdo a esta teoría la colocación de agujas de acupuntura y su posterior estimulación en los puntos energéticos acupunturales produce señales de tacto, presión o dolor «fino» trasmitidas por las fibras A beta que son rápidas, este estímulo es conducido a la sustancia gelatinosa de Rolando ( láminas II y III ) del asta dorsal de la médula espinal, excitándola y produciendo inhibición de la primera célula trasmisora del tracto espinotalámico (célula T), bloqueando la transmisión del impulso doloroso o cerrando la compuerta. El estímulo doloroso es conducido por las fibras A delta y C que son fibras finas y más lentas, este al llegar al asta dorsal de la médula espinal es bloqueado no produciéndose su transmisión al cerebro.

Es necesario señalar que el umbral de respuesta de las fibras A beta es menor que el umbral de respuesta de las fibras A delta y C, por lo que el nivel de estimulación debe estar por encima del umbral de las fibras A beta que se corresponde con el umbral de calambre, pero por debajo del umbral de las fibras A delta y C que se correspondería con el umbral de dolor, denominándose al área entre ambos umbrales zona terapéutica específica.

La acupuntura produce una serie de cambios a lo largo de las vías ascendentes del dolor y del sistema de inhibición descendente, por este mecanismo una de las áreas más afectadas es la activación inter neuronal en la médula espinal, la activación de estas neuronas previene la conducción de mecanismos de intensificación del dolor a la corteza. Además, la acupuntura activa el núcleo magno del rafe y estimula al hipotálamo atenuando la percepción dolorosa.

\section{Teoría embriológica de los dermatomas}

En las primeras etapas del desarrollo, el embrión (todavía plano) se cierra adquiriendo una forma tubular. A partir de esta estructura tubular, para un segmento dado de piel corresponde un órgano, y el ser humano adulto sigue conservando esta relación, por lo que estimulando distintos puntos en la piel podemos actuar sobre la función orgánica. Fisiológicamente, la inervación sensitiva de la piel confluye a un mismo nivel medular con la inervación de un determinado órgano. Esta disposición anátomo- funcional permite que el estímulo generado por la estimulación acupuntural desde la piel, al mismo nivel medular que la función orgá- nica que necesitamos modular. (Sussamann, 1989).

Una de las propiedades elementales del Sistema Nervioso, la plasticidad neuronal indica que las conexiones (sinapsis) interneuronales no son estables y constantes, sino que varían permanentemente en relación con los estímulos que proyectan. Las repetidas sesiones de acupuntura establecen un nuevo circuito neuronal, un nuevo «neuromatrix» anulando el circuito original del dolor. (El dolor crónico desarrolla un circuito del dolor que a medida que se prolonga en el tiempo es más difícil eliminarlo, pierde lentamente sus «características plásticas» para transformarse en un circuito rígido, una autopista de dolor. Sensibilización Central o Wind Up.

Todos estos mecanismos actúan asociados en diferentes niveles potenciando el efecto analgésico. Pero es importante destacar que la acupuntura usa mecanismos biológicos que ya, existen en nuestro organismo, no quita ni agrega energía, la canaliza y la modula mediante los mecanismos antes detallados. Los principios filosóficos-energéticos de la acupuntura son totalmente compatibles con los fundamentos neurofisiológicos actuales. De todas maneras aún falta mayor investigación en los mecanismos de acción de acupuntura. Cuando se realizan estímulos de baja frecuencia y alta intensidad se libera a nivel del eje hipotálamo-hipofisario beta endorfinas y ACTH (hormona adrenocorticotropa) mientras que en el cerebro medio y la médula espinal sólo encefalinas. Sin embargo, cuando se invierte la intensidad y frecuencia de la estimulación solamente se libera a nivel del cerebro medio y la médula espinal encefalina y serotonina a frecuencias inferiores a los $100 \mathrm{~Hz}$, los estímulos superiores ha demostrado la liberación de dinorfina y GABA en el líquido cefalorraquídeo.

Existen varias sustancias neurotransmisoras que intervienen en la transmisión del estímulo doloroso como la sustancia P, serotonina, ácido gamma aminobutírico (GABA) y noradrenalina entre otras, las que son modificadas por la acupuntura interfiriendo en la conducción de dicho estímulo.

Es conocido que los aferentes primarios que contienen sustancia P median los impulsos nociceptivos sobre todo los referidos a los estímulos de presión y los químicos, no así a los térmicos. Al producirse una disminución de la sustancia P como ocurre cuando se emplea la acupuntura, se produce una elevación del umbral doloroso, el papel funcional de la misma a nivel supramedular está aún en discusión.

La sustancia P en el nivel medular está involucrada en la 
transmisión del impulso doloroso con influencia en la despolarización postsináptica, así, como también con la modulación del dolor a través de mecanismos de inhibición presináptico y postsinápticos que involucran al GABA y facilita la analgesia acupuntural bloqueando los mecanismos de regulación postsináptica a modo de retroalimentación negativa que se refuerzan a través de las vías serotoninérgicas de inhibición.

La serotonina desempeña una función importante en el control del dolor crónico, mientras que la noradrenalina desempeña alguna función en el manejo del dolor agudo. Expone que la vía serotoninérgica en el rafe dorsal desempeña un importante rol en la analgesia por electro acupuntura a elevadas frecuencias de estimulación, Por la variabilidad interpersonal en la respuesta al dolor y en la analgesia por acupuntura, algunos autores como plantean que el genotipo de las personas así como la influencia de factores ambientales pueden ser de gran importancia en predecir qué pacientes serán beneficiados por esta modalidad analgésica.

\section{Neuro fisiología de la acupuntura}

La base de la acupuntura se expresa en el aforismo: "bu tong ze tong, tong ze bu tong", que significa "flujo libre: no hay dolor; no flujo libre: hay dolor". En otras palabras, cualquier tipo de dolor representa una obstrucción en el flujo normal de Qi o fuerza vital. La acupuntura mueve el Qi, restaurando el flujo libre. La selección de los puntos se basa en información obtenida a través de cuatro exámenes previos los cuales implican mirar, escuchar, preguntar y palpar. Una de las características únicas de la medicina clínica china es que los cuatro exámenes se actualizan continuamente, incluso dentro de una misma sesión.

Posterior aplicación de las agujas y otras técnicas tales como las ventosas o la moxibustión, un área del cuerpo puede ser palpada de nuevo, para evaluar si la temperatura, la textura, o la sensación de opresión o dolor han cambiado.

\section{Indicaciones de la acupuntura}

Se emplean la acupuntura China en las siguientes afecciones:

(World Health Organization, 1990)

Sistema Nervioso:

- Cefalea, Migraña, Dolores en general.

- Neuralgia (Trigémino, Intercostal) Ciática.
Parálisis (Facial, Hemiplejia, Hemiparesia)

- Disfuncionales Psicosexuales: Impotencia, Eyaculación precoz, Frigidez.

- Psicológico, Emocional: Stress, Ansiedad, Depresión, Insomnio.

Reumáticas: Artritis, Osteo artrosis, Fibromialgia.

Osteomuscular:

Dolor cervical, Tortícolis, Hombro doloroso.

Lumbago, Hernia discal (no calcificada)

Circulatorio:

Mala circulación, Calambres musculares.

Adormecimiento distal (mano y pie)

Neuropáticos: Diabético, Herpes zoster.

Alérgico: Rinitis, Sinusitis, Urticaria.

Ginecológico: Hiperémesis gravídica.

Metabólico: Anorexia, Bulimia.

Adicciones: Tabaco, Alcohol, Drogas, etc.

\section{CONCLUSIÓN}

1. El concepto Occidental referente a la Acupuntura China es que esta terapia ancestral estimula las terminaciones nerviosas y consecuente liberación de neurotransmisores. Este estímulo de las fibras nerviosas se da en los músculos, fascias, tendones o tejido peri articular y óseos, enviando impulsos a la médula espinal y activando varios centros en el encéfalo para la liberación de los neuro transmisores que ejercen un efecto homeostático en todo el cuerpo. A medida que avanza las investigaciones se conocen mejor estos mecanismos y se tiene una mayor y mejor comprensión de la cultura milenaria china, su filosofía y los misterios de esta ancestral técnicas de la Acupuntura. Así, las primeras comunicaciones entre científicos chinos se presentaron como las interrelaciones del Yin y Yang, lo que hoy podríamos perfectamente explicar como el Sistema Nervioso Simpático y Parasimpático, permitiéndonos una visión más académica de esta medicina milenaria.

\section{REFERENCIAS BIBLIOGRÁFICAS}

- Cabana, J. y Ruiz, R. (2004). Analgesia por acupuntura. Cuba: Revista Cubana de Medicina Militar.

- Goldberg, J. (1999). Las endorfinas: anatomía de un descubrimiento científico.

- Melzack, R. \& Wall, P. (1965). Pain mechanisms: a new theory. Science 150:197-210. 
- Nogier, F. (1954). Treatise of Auriculotherapy, Maisonneuve, Moulins-les-Metz. France.

- Soulié de Morant, G. (1990). Acupuntura. Buenos Aires, Argentina: Editorial Panamericana.

- Sussamann, D. (1989). Qué es la acupuntura. Que puede curar, Como actúa. Argentina: Editorial KIER S.A.

- Voll, R. \& Werner, F. (1978). Manual de Electroacupuntura. Editorial Literaria de Medicina, S. L. Stuttgart. Cuarta edición.

- World Health Organization (1990). Report of the Working Group on Auricular Acupuncture Nomenclature. Lyon, France. 